\title{
Oxidative Stress and Human Psoriasis Diseases
}

\author{
Asrat Endrias ${ }^{1}$, Menakath Menon ${ }^{2}$, Mihretu Wodeyes ${ }^{3}$, Tamrat Abebe ${ }^{4}$, Mohammed Mehdi ${ }^{2}$, \\ Ezra Belay ${ }^{1}$, Daniel Seifu ${ }^{2}$ \\ ${ }^{1}$ Department of Biochemistry, Mekelle University, Mekelle, Ethiopia \\ ${ }^{2}$ Department of Biochemistry, Addis Ababa University, Addis Ababa, Ethiopia \\ ${ }^{3}$ Department of Dermatology, Addis Ababa University, Addis Ababa, Ethiopia \\ ${ }^{4}$ Department of Microbiology, Parasitology and Immunology, Addis Ababa University, Addis Ababa, Ethiopia
}

Email address:

endasrias@gmail.com (A. Endrias),menakath.menon@aau.edu.et(M. Menon),mihertuwg@yahoo.com (M. Wodeyes), tamrat.abebe@aau.edu.et (T. Abebe), mohammed.mehdi@aau.edu.et(M. Mehdi), aga.bely@gmail.com (E. Belay), daniel.seifu@aau.edu.et (D. Seifu)

${ }^{*}$ Corresponding author

\section{To cite this article:}

Asrat Endrias, Menakath Menon, Mihretu Wodeyes, Tamrat Abebe, Mohammed Mehdi, Ezra Belay, Daniel Seifu. Oxidative Stress and Human Psoriasis Diseases. American Journal of Laboratory Medicine. Vol. 5, No. 4, 2020, pp. 118-124. doi: 10.11648/j.ajlm.20200504.16

Received: July 15, 2020; Accepted: July 28, 2020; Published: August 10, 2020

\begin{abstract}
Psoriasis is a chronic inflammatory skin disease characterized by the immune system activation due to genetic and environmental factors that leads to the development of skin lesions. Reactive oxygen species produced as a result of skin inflammation may cause disorders of the antioxidant defense systems and increased oxidative stress in psoriasis which was proposed to have a consequent function in psoriasis. The study was planned to evaluate oxidative stress by measuring Total Oxidant Capacity, Total Anti-oxidant capacity and Oxidative Stress Index in psoriatic patients. Methodology: A comparative cross-sectional study design was used. A total of 90 participants between age of 18-65 years comprising 45 newly diagnosed psoriatic patients and 45 sex and age matched control groups were enrolled in the study. Result: The present study showed significantly increased levels of serum Total Oxidant Capacity $(p<0.001)$ as well as Oxidative Stress Index $(p<0.001)$ in psoriasis patients as compared to controls and positively correlated with severity and duration of the disease. Total Antioxidant capacity levels were significantly $(\mathrm{p}<0.001)$ lower in patients than in controls and negatively correlated with severity and duration of the disease. Conclusion: These results provide evidences regarding the role of increased reactive oxygen species with decreased antioxidant activity in psoriatic patients.
\end{abstract}

Keywords: Total Oxidant Capacity, Total Antioxidant Capacity, Oxidative Stress Index, Psoriasis

\section{Introduction}

Skin problems are generally the most common diseases seen in primary care setting all over the globe and its prevalence ranges from $20-50 \%$ in developing countries. Compared with other diseases, skin diseases have a lower mortality rate but Patients of skin disease always experience physical, emotional and socio-economic embarrassment in the society [1].

Psoriasis is one amongst these notorious auto-immune disorder having deep psychological and social impacts [2]. Psoriasis is non- contagious skin disease affecting both sexes equally, and can occur at any age, although it most commonly appears for the first time between the ages of 15 and 25 years [3, 4]. Worldwide psoriasis is affecting, as presumed, approximately $120-180$ million people. The population prevalence of psoriasis has been reported to range from $2 \%$ to $3 \%$. Around 150,000 new cases of psoriasis are reported annually. Studies in developed countries have reported higher prevalence rates of on average about $4.6 \%$ [5].

In Ethiopia, according to some studies, up to $80 \%$ of people have one or more skin diseases. Information on the prevalence of psoriasis in Ethiopia is extremely limited; however, data on the incidence of psoriasis at a specified clinic or hospital over a defined period of time are more 
frequently available. While the absolute incidence rate at a clinic may fail to reflect the local prevalence of psoriasis, the relative rates of psoriasis incidence, obtained from clinics were retrieved and reviewed. A total of 2342 dermatologic cases were diagnosed at ALERT pathology laboratory from January 2007 to December 2010 which represent a wide range of inflammatory, infectious, and neoplastic diseases. During this period out of 632 inflammatory dermatoses 43 were diagnosed with psoriasis [7].

The skin is a potential target for oxidative injury, as it is continuously exposed to UV radiation and other environmental stresses generating reactive oxygen species (ROS) [8]. ROS mediated oxidative damage involves a vast number of biological molecules since it causes lipid peroxidation, DNA modification, and secretion of inflammatory cytokines [1].

The pathogenesis of psoriasis is not fully understood but evidence suggests that there is a strong genetic component which is mediated by abnormal $\mathrm{T}$ lymphocyte function [9]. Oxidative stress is believed to be a key factor in the pathogenesis of psoriasis. Studies have suggested the involvement of increased ROS levels in psoriasis pathogenesis. Increased ROS generation by infiltrated leukocytes into psoriatic lesions is accompanied by substantial biomolecular damage, like psoriatic skin lesions containing oxidized LDL [10].

In order to cope with constant and excess efflux of damaging reactive metabolites, the skin has developed several lines of defense. Deficiencies in any of the antioxidant defense system can cause a reduction in the total antioxidant status (TAS) of an individual (Attwa and Swelam 2011). However, inadequate antioxidant protection or excess ROS production creates a condition known as oxidative stress, contributing to the development of cutaneous disease and disorders [4].

However it is questionable whether the observed abnormalities are responsible for the onset of psoriasis, or resultant from ongoing pathologic process. Therefore, the hypothesis of an imbalance between oxidants and antioxidants in psoriasis and its role in the pathogenesis of the disease still remain to be elucidated.

As far as the present investigations are concerned, no previous study is done in Ethiopia about the status of oxidative stress among psoriatic patients. Therefore, this research was aimed to evaluate the total oxidant-antioxidant capacity and oxidative stress index among newly diagnosed psoriatic patients in Alert Hospital, Addis Ababa, Ethiopia.

\section{Methods and Materials}

A comparative cross-sectional study was conducted between September 2015 and January, 2016 at ALERT Hospital Addis Ababa, Ethiopia. Accordingly, a total of 90 participants between age of 18-65 years comprising 45 newly diagnosed psoriatic patients who had given informed written consent and 45 sex and age matched control groups were enrolled in the study.
Psoriasis patients with no history of any drug therapy for last two months were included in the study. Patients with past or present history of any disease like diabetes, neoplastic diseases, liver and kidney disorders, psychological diseases and infections; those with immune-suppressing conditions or familial hypercholesterolemia; and those with a history of major surgery which could affect oxidative stress were excluded from the study. The severity of the psoriasis was determined by Psoriasis Area Severity Index (PASI) score and patients were grouped as mild, Moderate and sever psoriasis. Clinical severity of psoriasis was determined according to the Psoriasis Area Severity Index (PASI) score. According to PASI score patients were classified into 3 subgroups: (1) Mild group: 22 patients with PASI score less than 15. (2) Moderate group: 15 patients with PASI score between 15 and 25. (3) Severe group: 8 patients with PASI score more than 25 .

\subsection{Blood Sample Collection Procedure}

Data were collected by professional laboratory technologists, nurses and physicians under the supervision of principal investigator. Under preceding instructions psoriatic patients and healthy controls were checked regarding fasting by interview on the morning of the examination by the physician or Nurse. Pertinent information was obtained from standardized clinical files designed for the program and extracted from it using a questionnaire and other demographic details were subsequently collected.

Blood Samples

A total of 90 blood samples (45 psoriasis patients and 45 control groups) with a volume of $3-5 \mathrm{ml}$ were collected using serum separator tube (SST). Serum sample was harvested into Eppendorf tube after the blood samples were centrifuged at $4000 \mathrm{rpm}$ for five minutes then. Then, it was stored in $80^{\circ} \mathrm{C}$ deep freezer until the analysis for two weeks.

\subsection{Chemicals and Equipment's Used}

\section{Total Oxidant Status (TOS) Assay Principle}

It was determined based on the principles of Erel [11]. In this process oxidants present in the sample oxidize the ferrous particle o-dianisidine complex to the ferric particle. The oxidation reaction is upgraded by glycerol molecules, which are richly present in the reaction medium. A colored compound is formed when the ferric ion reacts with xylenol orange in an acidic medium. The color strength, which can be measured spectrophotometrically at $560 \mathrm{~nm}$ wave length, is correlated to the total quantity of oxidant molecules present in the plasma. The assay is aligned with hydrogen peroxide and the outcomes are expressed as far as $\mu$ molar hydrogen peroxide equivalent per liter $\left(\mu \mathrm{mol} \mathrm{H}_{2} \mathrm{O}_{2} \mathrm{Eq} / \mathrm{l}\right)$ [11].

\subsection{Total Antioxidant Capacity (TAC) Assay Principle}

It was determined based on the principles of Koracevic [12]. In this technique, the hydroxyl radical, the most powerful natural radical, is generated by the Fenton reaction 
and it responds with the colorless substrate O-dianisidine to create the dianisyl radical, which is splendid yellowishbrown in color. Upon the addition of sample, the oxidative responses started by the hydroxyl radicals present in the reaction are scavenged by the antioxidant agents present in the sample, keeping the color change and consequently giving a viable estimation of TAC [12]. The test results were expressed as mmol Trolox Eq/l.

\subsection{Determination of Oxidative Stress Index (OSI)}

It was calculated based on the method of Erel [11]. The proportion of TOS to TAC is acknowledged as the oxidative stress index (OSI). For estimation, the subsequent unit of TAC is changed over to $\mathrm{mmol} / \mathrm{l}$, and the OSI value is computed.

$$
\text { OSI (subjective unit) }=\operatorname{TOS}\left(\mu \mathrm{mol} \mathrm{H}_{2} \mathrm{O}_{2} \mathrm{Eq} / \mathrm{l}\right) / \mathrm{TAC}^{*} 100
$$
(mmol Trolox Eq/l) [11].

\subsection{Data Processing and Software Used in Statistical Analysis}

All data's were checked, cleared and fed into Epi-data (version 3.5.1, 2008) and then exported to SPSS (version 22.0, 2012, America) software for statistical analysis. Descriptive analysis, Spearman correlation, and linear regression, independent sample T-test, one way ANOVA followed by post hoc analysis were used for this study. All data's were expressed in mean $\pm \mathrm{SD}$ and $\mathrm{P} \leq 0.05$ was considered as a statistically significant.

\subsection{Ethical Consideration}

The study was ethically cleared from ethical committee of the Department of Biochemistry (DRERC) with a protocol number of 04/15 DRERC, and ALERT/AHRI ethics review committee /AAERC/ with a protocol number P011/15.

All participants in study had information on study. A consent form was prepared with detailed explanation of objectives, risks, and benefits to the study subjects and the assurance of confidentiality of responses were given to participants. Data were collected after obtaining informed consent and agreement from the patients under study.

Sample collection was performed by trained health professionals following ethical steps and procedures. Only patients who had signed the informed consent were enrolled in the study. Strict confidentiality of all information collected was maintained and all protocols of safety were followed throughout the study. Assurances were given to the participants on confidentiality of collected data.

\section{Results}

\subsection{Demographic Characterization}

Among 90 participants, 45 of them were psoriatic patients (24 males and 21 females) with mean age $39.69 \pm 12.67$ and BMI of $21.84 \pm 2.63$. The rest of 45 were age-and sexmatched healthy subjects as a control group (26 males and 19 females) with mean age $38.53 \pm 12.05$ and BMI of $23.38 \pm$ 2.79. The mean score for clinical severity was $20.08 \pm 16.94$. Psoriasis patients were further graded according to the PASI as, mild (22/45 patients), moderate (15/45 patients) and severe ( $8 / 45$ patients). Disease duration of the patients ranged from 3 weeks to 5 years with mean $2.39 \pm 1.61$ (Table 1). The type of psoriasis 38 or $84 \%$ psoriatic patients have Plaque type of lesions. Plaque psoriasis lesions are well defined, raised, erythematous with a sharply defined edge associated with silvery white scales.

Table 1. Demographic characteristics and clinical features of psoriatic patients and healthy Controls.

\begin{tabular}{lll}
\hline & $\begin{array}{l}\text { Healthy Controls } \\
(\mathbf{n = 4 5 )}\end{array}$ & $\begin{array}{l}\text { Psoriatic } \\
\text { Patients (n=45) }\end{array}$ \\
\hline Sex $[\mathrm{M} / \mathrm{F}]$ & $26 / 19$ & $24 / 21$ \\
Age $[\mathrm{years}]$ & $38.53 \pm 12.05$ & $39.69 \pm 12.67$ \\
Height $[\mathrm{m}]$ & $1.60 \pm 0.09$ & $1.62 \pm 0.08$ \\
Weight $[\mathrm{kg}]$ & $60.13 \pm 8.86$ & $57.53 \pm 9.36$ \\
Body mass index $\left[\mathrm{kg} / \mathrm{m}^{2}\right]$ & $23.38 \pm 2.79$ & $21.84 \pm 2.63$ \\
PASI & & $20.08 \pm 16.94$ \\
Mild & & $22(48 \%)$ \\
Moderate & & $15(33 \%)$ \\
Sever & & $8(17 \%)$ \\
Type of lesion & & \\
Plaque & & $38(84 \%)$ \\
Erythema & & $1(2 \%)$ \\
Papules & & $3(6 \%)$ \\
Pustule & & $3(6 \%)$ \\
Psoriasis duration [years] & & $2.39 \pm 1.61$ \\
\hline
\end{tabular}

Results presented as mean \pm SD and frequency (\%) (Except male: female ratio)

\subsection{Estimated Biochemical Parameters}

Total Oxidant- Antioxidant Between Psoriasis and Health Control

Total oxidant capacity (TOC) $(13.94 \pm 2.28)$ and oxidative stress index (OSI), (1.32 \pm 0.59$)$ were significantly higher in psoriatic patients compared to control subjects (11.60 $\pm 1.43)(\mathrm{p}<0.01)$. On the other hand, the levels of Total Antioxidant capacity (TAC) were significantly decreased in psoriatic patients $(1.15 \pm 0.21)$ compared to normal healthy controls $(1.36 \pm 0.16)(\mathrm{p}<0.01)$ (Table 2).

Table 2. TOC, TAC and OSI levels in patients versus controls.

\begin{tabular}{lll}
\hline Parameter & Healthy Contorts $(\mathbf{n = 4 5})$ & Psoriatic Patients $(\mathbf{n}=\mathbf{4 5})$ \\
\hline TOC $\left[\mathrm{mmol} \mathrm{H}_{2} \mathrm{O}_{2}\right.$ eqv./l] & $11.60 \pm 1.43$ & $13.94 \pm 2.28$ \\
TAC [mmol Trolox eqv./l] & $1.36 \pm 0.16$ & $1.15 \pm 0.21$ \\
OSI $[\mathrm{AU}]$ & $0.86 \pm 0.15$ & $1.32 \pm 0.59$ \\
\hline
\end{tabular}

Results presented: as mean \pm SD, TAC - Total Antioxidant capacity, TOC - Total Oxidant capacity, OSI - Oxidative Stress Index. 
Present study shows that, TOC and OSI levels were significantly $(\mathrm{P}<0.01)$ increased in moderate psoriatic patients than the patients presenting with mild psoriasis, whereas, severe psoriatic patients exhibited significant increase in TOC and OSI $(\mathrm{P}<0.001)$ as compared to moderate psoriatic patients.
Furthermore, TAC activity in moderate psoriatic patients were significantly $(\mathrm{P}<0.01)$ lower than the patients presenting with mild psoriasis, whereas, severe psoriatic patients exhibited significant decrease in TAC $(\mathrm{P}<0.01)$ as compared to moderate psoriatic patients (Table 3).

Table 3. Changes in levels of TOC, TAC and OSI within different groups of psoriasis patients.

\begin{tabular}{|c|c|c|c|c|}
\hline \multirow{2}{*}{ Parameters } & \multirow{2}{*}{ Control $(n=45)$} & \multicolumn{3}{|c|}{ Psoriatic patients $(n=45)$} \\
\hline & & Mild (n=22) & Moderate $(n=15)$ & Sever $(n=8)$ \\
\hline TOC [mmol $\mathrm{H}_{2} \mathrm{O}_{2}$ eqv./l] & $11.60 \pm 1.43$ & $12.47 \pm 1.11^{\mathrm{blc} 2}$ & $14.03 \pm 1.52^{\mathrm{a} 2 \mathrm{c} 2}$ & $17.81 \pm 0.62^{\mathrm{a} 2}$ \\
\hline TAC [mmol Trolox eqv./1] & $1.36 \pm 0.16$ & $1.29 \pm 0.52^{\mathrm{b} 1 \mathrm{c} 2}$ & $1.14 \pm 0.04^{\mathrm{a} 2 \mathrm{c} 2}$ & $0.76 \pm 0.16^{\mathrm{a} 2}$ \\
\hline OSI [AU] & $0.86 \pm 0.15$ & $0.96 \pm 0.10^{\mathrm{b} 1 \mathrm{c} 2}$ & $1.26 \pm 0.16^{\mathrm{a} 2 \mathrm{c} 2}$ & $2.43 \pm 0.57^{\mathrm{a} 2}$ \\
\hline
\end{tabular}

Results presented as mean $\pm \mathrm{SD}$, TAC - Total Antioxidant capacity, TOC - Total Oxidant capacity, OSI -Oxidative stress index; ${ }^{a}$ compared to control, bompared to moderate, ${ }^{\mathrm{c}}$ compared to severe; ${ }^{1} \mathrm{p}<0.01,{ }^{2} \mathrm{p}<0.001$

\subsection{Psoriasis Disease Duration and Changes in Total Oxidant- Antioxidant Status}

Disease duration of psoriatic patients were ranged from 3 weeks to 5 years with mean $2.39 \pm 1.61$ (Table 1) and further categorized into $<1,1-3$ and $>3$ years. Psoriatic patients with a year duration greater than 3 years have showed a significant increase in TOC and OSI $(P<0.001)$ and with a decreased TAC levels when compared to patients with a year duration less than a year and patients between 1 to 3 years (Table 4)

Table 4. Psoriasis disease duration and changes in levels of TOC, TAC and OSI in psoriatic patients.

\begin{tabular}{llll}
\hline \multirow{2}{*}{ Parameters } & \multicolumn{3}{l}{ Psoriasis disease duration [years] } \\
\cline { 2 - 4 } & $<\mathbf{1}$ & $\mathbf{1 - 3}$ & $>\mathbf{3}$ \\
\hline TOC [mmol H $\mathrm{O}_{2}$ eqv./l] & $12.41 \pm 1.23^{\mathrm{cl}}$ & $12.39 \pm 0.87^{\mathrm{cl}}$ & $15.86 \pm 1.89^{[\mathrm{ba}] 1}$ \\
TAC [mmol Trolox eqv./l] & $1.29 \pm 0.05^{\mathrm{cl}}$ & $1.25 \pm 0.08^{\mathrm{c} 1}$ & $0.98 \pm 0.21^{[\mathrm{ba}] 1}$ \\
OSI [AU] & $0.96 \pm 0.11^{\mathrm{cl}}$ & $0.1 \pm 0.08^{\mathrm{cl}}$ & $1.75 \pm 0.68^{[\mathrm{ba}] 1}$ \\
\hline
\end{tabular}

Results presented as mean $\pm \mathrm{SD}, \mathrm{TAC}-$ Total Antioxidant capacity, TOC - Total Oxidant capacity, OSI -Oxidative stress index; ${ }^{\mathrm{a}}$ compared to $<1$, ${ }^{\mathrm{b}}$ compared to $1-3,{ }^{\mathrm{c}}$ compared to $>3 ;{ }^{1} \mathrm{p}<0.001$

\subsection{Correlations Between Total Oxidant-Antioxidant in Psoriatic Patients}

The levels of TOC showed a significantly negative correlation with with TAC association $(p<0.001)$. On the other hand TOC showed a significantly positive correlation with levels of OSI $(\mathrm{p}<0.001)$ association in psoriatic patients (Table 5).

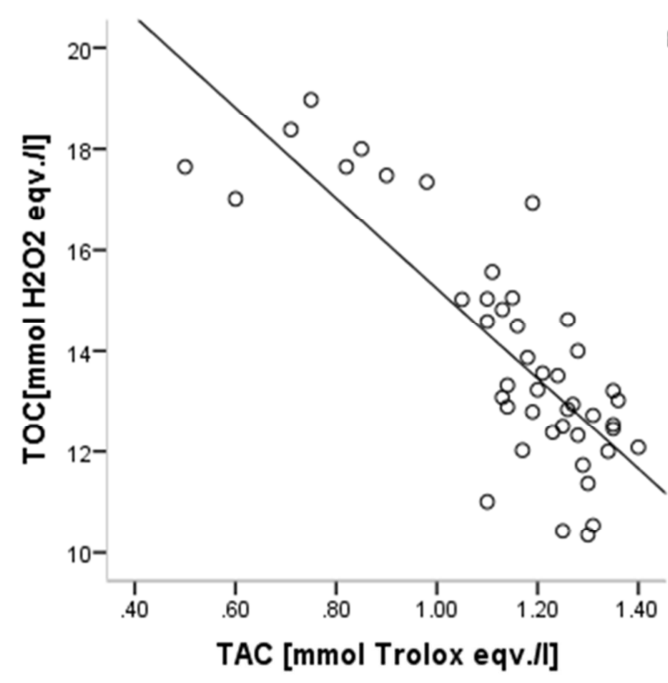

(a) $\mathrm{r}=-0.81 \mathrm{P}=<0.001$
Table 5. Correlations between TOC levels and TAC, OSI levels in psoriatic patients.

\begin{tabular}{lll}
\hline Parameter & R & P-value \\
\hline TOC and TAC & -0.81 & $<0.001$ \\
TOC and OSI & 0.86 & $<0.001$ \\
\hline
\end{tabular}

Total Antioxidant capacity, TOC - Total Oxidant, OSI - Oxidative Stress Index, ${ }^{* *} \mathrm{p}<0.001$, Results were expressed in pearson correlation coefficient $(-)$ negative and $(+)$ Positives correlation

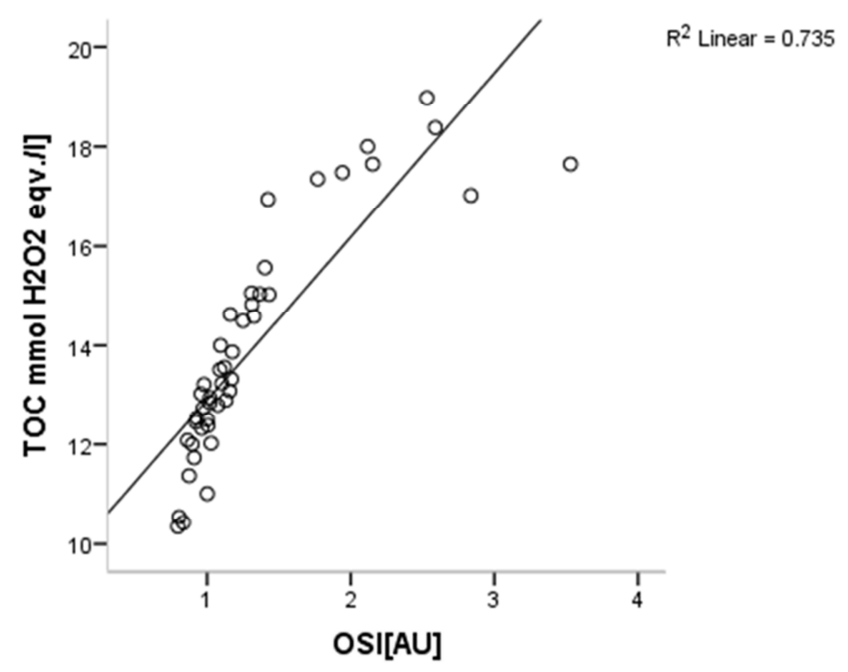

(b) $\mathrm{r}=086 \mathrm{p}=<0.001$

Figure 1. Regression fit of (a) TOC VS TAC and (b) TOC VS OSI. 


\subsection{Correlations Between Total Oxidant-Antioxidant and Severity of Disease}

It was found a negative correlation between TOC and TAC but it was not significant $(\mathrm{p}>0.05)$ among mild, moderate and severs psoriatic patients. Conversely a positive correlation was found between TOC and OSI among the three groups with significant correlation in mild and moderate $(\mathrm{p}<0.001)$ whereas, severe psoriatic patients exhibited insignificant $(\mathrm{P}>0.05)$ correlation (Table 6).

Table 6. Correlations between TOC levels and TAC, OSI levels among the different groups of psoriasis patients.

\begin{tabular}{llll}
\hline & Mild & Moderate & Severe \\
\hline TOC and TAC & $-0.22^{\mathrm{a}}$ & $-0.047^{\mathrm{a}}$ & $-0.018^{\mathrm{a}}$ \\
TOC and OSI & $+0.927^{\mathrm{b}}$ & $+0.949^{\mathrm{b}}$ & $+0.065^{\mathrm{a}}$ \\
\hline
\end{tabular}

Results were expressed in pearson correlation coefficient (-) negative and $(+)$ Positives correlation among variables a $-\mathrm{p}>0.05,{ }^{\mathrm{b}} \mathrm{p}<0.001$

\section{Discussion}

Skin cells are a major target of oxidative stress mainly due to ROS originating from the environment and skin metabolism itself. Although endogenous antioxidants attenuate the harmful effects of ROS, increased or prolonged presence of free radicals can override ROS defense mechanisms and mediate numerous cellular responses that contribute to the development of a variety of skin disorders, including psoriasis [13]

Overproduction of ROS during the inflammatory process in psoriasis may result in oxidative stress leading to damage of cellular structures such as lipids, proteins and DNA and interference with production of cytokines such as IL-1 and TNF- $\alpha$. It is also responsible for a decrease in the cAMP/cGMP ratio leading to epidermal hyperproliferation $[14,15]$.

The present work showed a significantly increased concentration of TOC in psoriatic patients (13.94 \pm 2.28$)$ compared to healthy controls $(11.60 \pm 1.43)(\mathrm{p}<0.001)$. This significantly elevated TOC could be due to high free radical load and insufficient antioxidant which consequently indicates a high oxidative stress condition in psoriatic patients. In concordance with this study, Sürücü et al, [16], Karababa et al., [17], Gabr et al., [18] and Hashemi et al., [19] have reported increased TOC levels in psoriatic patients as compared to healthy controls.

We also found that the concentrations of serum TOC were increased with severity of the psoriasis. The values of TOC were significantly higher in, moderate psoriatic patients $(14.03 \pm 1.52)$ than mild psoriatic patients $(12.47 \pm 1.11)$ $(p<0.001)$ as well as severe psoriatic patients $(17.81 \pm 0.62)$ than moderate psoriatic patients $(\mathrm{p}<0.001)$ respectively and also the research considered increased TOC levels as psoriasis year duration increases $(P<0.001)$ thereby confirming that the load was decidedly higher with the progression of psoriasis (Table 4). In very early phase of developing psoriasis lesions, macrophages were seen within the epidermis followed by lymphocytes. During subsequent development neutrophils began to appear between the upper layers forming pockets (Micro-abscesses). Neutrophil migration into the epidermis was most pronounced in active disease and occurred in a rhythmic pattern [20]. These presumptions were well supported by findings of Attwa and Swelam [21] and Jyoti et al., [22].

Skin cells are equipped with both enzymatic [superoxide dismutase (SOD), catalase (CAT), glutathione peroxidase (GP)] and non-enzymatic mechanisms (vitamin C, vitamin E, carotenoids, thiol antioxidants, flavinoids, selenium and others) to deal with the harmful effects of ROS [23]. Antioxidants can protect the epidermis from the events that contribute to epidermal toxicity and diseases. Deficiencies in any of the antioxidant defense system can cause a reduction in the total antioxidant status of an individual [10].

The results of this study showed statistically significant $(p<0.001)$ differences in TAC values $(1.15 \pm 0.21)$ between psoriatic patients and healthy controls $(1.36 \pm 0.16)$ (Table 2$)$. This is in concordance with the studies of Sürücü et al,. [16], Karababa et al., [17], Gabr et al.,[18] and Hashemi et al., [19] which suggest that antioxidant defense mechanisms are exhausted in psoriatic patients.

The decreased TAC may be possibly due to depressed state of antioxidant system or due to the exaggerated inflammatory processes and oxidative stress in these patients. Antioxidants prevent oxidative injury of structural lipids and proteins contributing to barrier integrity, which is essential for healthy skin condition. This suggests that cellular redox environment plays a pivotal role in skin homeostasis and that skin disease could result from an imbalance between pro-oxidant and antioxidant stimuli [24].

One important line of defense is a system of enzymes, including glutathione peroxidase, superoxide dismutase (SOD) and catalase (CAT) which decreases the concentration of the most harmful oxidants. During oxidative stress there can be the overload of superoxide radicals in the psoriasis [25]. Since, the natural antioxidant defense system SOD limits the development of inflammatory and immune processes, the tendency to decreased SOD activity in psoriasis patients is one of the reasons for the aggravated dermatological status. This over formation of the superoxide radical also was proposed to inhibit the activity of catalase [26].

However, in a study performed by Nassiri et al., [14] found no significant difference in serum TAC levels between patient cases and controls. They explained this by the younger age of Iranian patients and also because Iranian regular diet which contains more fruit and vegetable and less saturated fatty acid in comparison with western diets. Nevertheless in our study, we didn't find a strong correlation on the influence of age in serum TAC levels.

The present study serum TAC levels were decreased significantly in moderate psoriasis patients than mild psoriasis patients $(\mathrm{p}<0.001)$ whereas, severe psoriatic patients exhibited significant decrease in TAC activity $(\mathrm{P}<0.001)$ as 
compared to moderate psoriatic patients (Table 3 ).

Moreover, a decreased TAC levels was observed as psoriasis year duration increases $(\mathrm{P}<0.001)$ (Table 4). The low TAC level can be explained with excessive depletion of the antioxidant capacity caused by free radical elimination to reduce the detrimental effects of ROS and their products in order to maintain pro-oxidant-antioxidant homeostasis. Pujari et al [20] also found positive correlation between increased MDA, NO in serum of psoriatic patients with severity of psoriasis whereas negative correlation between decreased TAC with severity of psoriasis. The reduced level of TAC may reflect a decrease in the antioxidant capacity.

When the oxidant/antioxidant balance is tilted towards oxidants and oxidative stress arises, there is a significant negative correlation between the TAC and TOC values [27]. In the present investigation a negative correlation was demonstrated between the TAC and TOC, which is one of the scales indicating the severity of psoriasis. This corresponds to previously reported findings $[16,17]$. These findings may indicate the importance of the anti-oxidant system in the pathogenesis and severity of psoriasis.

Oxidative stress index (OSI), a proportional value between TAC and TOC, is a recently investigated parameter that is affected directly by oxidant and antioxidant status and that can present the degree of oxidative stress more clearly. Therefore, OSI may be a useful and practical parameter for evaluating oxidative injury in psoriatic patients [28].

The OSI estimated in this study was significantly $(\mathrm{p}<0.001)$ higher in psoriatic patients $(1.32 \pm 0.59)$ than in healthy controls $(0.86 \pm 0.15)$ (Table 2$)$. This indicates the exact degrees of imbalance of Oxidative stress towards the oxidant status. This finding is in keeping with most previously published findings as Gabr and Al-Ghadir [18] and Hashemi et al., [19] have reported high OSI values in psoriasis patients compared with control groups. On the basis of these results, we can deduce that a compromised antioxidant defense mechanism, accompanied by increased oxidant levels and OSI values in psoriasis patients, might play an important role in the pathogenesis of this disease.

Present study showed that there was statistically significant increase in OSI value in mild $(\mathrm{P}<0.01)$, moderate $(\mathrm{P}<0.01)$ and severe $(\mathrm{P}<0.01)$ psoriasis patients when compared to healthy controls. In addition, OSI in moderate psoriatic patients were significantly $(\mathrm{P}<0.01)$ lower than the patients presenting with mild psoriasis, whereas, severe psoriatic patients exhibited significant decrease in OSI $(\mathrm{P}<0.01)$ as compared to moderate psoriatic patients (Table 3 ) and also the research considered increased OSI as disease duration increases $(P<0.001)$ (Table 4). Similar results were reported by Sürücü et al., [16,], Gabr and Al-Ghadir [18]. This might be due to the prolonged release of excess ROS in the skin cells which can aggravate inflammatory injury and promote chronic inflammation. The decrease in TAC and increase in TOC was more pronounced in severe patients comparing to mild and moderate indicating that the antioxidants were nearly completely utilized to scavenge the superoxide free radicals.

\section{Conclusion}

The presented study concluded that oxidative stress in psoriatic patients indicated by increased TOC as well as OSI and decreased TAC levels when compared to healthy controls. Furthermore the study found worsened oxidant and antioxidant status according to the severity and duration of disease which may presumably be the causative factor in the pathogenesis of psoriasis. This should be noted in all future studies. These findings suggest that a weakened antioxidant defense system may play a significant role in the increased oxidative stress found in psoriatic patients. Therefore, it is possible to conclude that increase of oxidative stress in psoriatic patients is due to increase in severity and duration of the disease. Moreover, this study also supports the possibility of involvement of oxidative stress in pathogenesis of psoriasis.

Therefore, estimating the status of TOC, TAC and ISO in psoriatic patients plays a critical role as an effective biomarker of ROS and may have even greater potential to develop novel strategies for diagnosis, prognosis and treatment of psoriasis patients.

\section{Funding}

The authors are grateful to Addis Ababa University, College of Health sciences, School of Medicine for funding this research project.

\section{Conflict of Interest}

The authors declare that they have no competing interests.

\section{Acknowledgements}

We would like to acknowledge the contributions of study participants, Biochemistry Department of Addis Ababa University and Alert Hospital, Addis Ababa, Ethiopia.

\section{References}

[1] Baur, B., Sarkar, J., Manna, N. and Bandyopadhyay, L.,. The pattern of dermatological disorders among patients attending the skin OPD of a tertiary care hospital in Kolkata. India. IOSR Journal of Dental and Medical Sciences (JDMS), 2013; 4 (3), pp. 04-09.

[2] Prasanth, D., Masram, P., Paul, R. and Anup, T. A systemic analysis on psoriasis. WSR to EKAKUSHTA, Pharma Science Monitor 5 (3) Supl-1 July-Sep 2014. 333-347.

[3] Chong, L. Is Psoriasis a Cutaneous Disease or Systemic Disease?. Medical Bulletin, 2010; 15 (11).

[4] Dilnawaz, M., Sadiq, S., Shaikh, Z., Aziz, H., Khan, S. A. and Jawad, B. Clinical audit: baseline Psoriasis Area and Severity Index (PASI) and Dermatology Life Quality Index (DLQI) assessment of psoriasis patients. Journal of Pakistan Association of Dermatologists, 2013; 23 (4), pp. 407-411. 
[5] Chandran, V. and Raychaudhuri, S. P. Geoepidemiology and environmental factors of psoriasis and psoriatic arthritis. Journal of autoimmunity, 2010; 34 (3), pp. J314-J321.

[6] Diallo, M.,. Psoriasis Epidemiology. Journal of Clinical Case Reports, 2012.

[7] Gimbel, D. C. and Legesse, T. B. Dermatopathology practice in Ethiopia. Archives of Pathology and Laboratory Medicine, 2013; 137 (6), pp. 798-804.

[8] Balato, A., Ayala, F., Schiattarella, M., Megna, M., Balato, N. and Lembo, S. Pathogenesis of psoriasis: the role of proinflammatory cytokines produced by Keratinocytes. INTECH Open Access Publisher, 2012.

[9] Mantovani, A., Gisondi, P., Lonardo, A. and Targher, G. Relationship between Non-Alcoholic Fatty Liver Disease and Psoriasis: A Novel Hepato-Dermal Axis? International journal of molecular sciences, 2016; 17 (2), p. 217.

[10] Wagener, F. A., Carels, C. E. and Lundvig, D. Targeting the redox balance in inflammatory skin conditions. International journal of molecular sciences, 2013; 14 (5), pp. 9126-9167.

[11] Erel, O. A new automated colorimetric method for measuring total oxidant status. Clinicalbiochemistry, 2005; 38 (12), pp. 1103-1111. https://doi.org/10.1016/j.clinbiochem.2005.08.008

[12] Koracevic D., Koracevic G., Djordjevic V., Andrejevic S. And Cosic V. Method for the measurement of antioxidant activity in human fluids. J. Clin Pathol, 2001; 54, 356-361.

[13] Zhou, Q., Mrowietz, U. and Rostami-Yazdi, M. Oxidative stress in the pathogenesis of psoriasis. Free Radical Biology and Medicine, 2009; 47 (7), pp. 891-905.

[14] Nassiri, S., Malekzad, F., Sarlak, M., Saeedi, M., Hedayati, M. A. H. D. I. and Qaisari, M. Interplay among antioxidants and oxidants in psoriasis. Iranian Journal of Dermatology, 2009; $12(2)$, pp. 56-59.

[15] Guven, B., Can, M., Genc, M. and Koca, R. Serum prolidase activity in psoriasis patients. Archives of dermatological research, 2013; 305 (6), pp. 473-476.

[16] Sürücü, H. A., Aksoy, N., Ozgöztas, O., Sezen, H., Yesilova, Y. and Turan, E. Prolidase activity in chronic plaque psoriasis patients. Advances in Dermatology and Allergology/Postępy Dermatologii i Alergologii, 2015; 32 (2), p. 82.

[17] Karababa, F., Yesilova, Y., Turan, E., Selek, S., Altun, H. and Selek, S.. Impact of depressive symptoms on oxidative stress in patients with psoriasis. Redox Report, 2013; 18 (2), pp. 5155 .

[18] Gabr, S. A. and Al-Ghadir, A. H. Role of cellular oxidative stress and cytochrome $c$ in the pathogenesis of psoriasis. Archives of dermatological research, 2012; 304 (6), pp. 451457.

[19] Hashemi, M., Mehrabifar, H., Daliri, M. and Ghavami, S. Adenosine deaminase activity, trypsin inhibitory capacity and total antioxidant capacity in psoriasis. Journal of the European Academy of Dermatology and Venereology, 2010; 24 (3), pp. 329-334.

[20] Pujari, V. M., Ireddy, S., Itagi, I. and Kumar, S. The Serum Levels of Malondialdehyde, Vitamin E and Erythrocyte Catalase Activity in Psoriasis Patients. Journal of clinical and diagnostic research: JCDR, 2014; 8 (11), p. CC14.

[21] Attwa, E. and Swelam, E. Relationship between smokinginduced oxidative stress and the clinical severity of psoriasis. Journal of the European Academy of Dermatology and Venereology, 2011; 25 (7), pp. 782-787.

[22] RS Jyothi, KS Govindswamy, K Gurupadappa - Psoriasis: An oxidative stress condition. J Clin Diagn Res, 2011.

[23] Şikar Aktürk, A., Özdoğan, H. K., Bayramgürler, D., Çekmen, M. B., Bilen, N. and Kiran, R. Nitric oxide and malondialdehyde levels in plasma and tissue of psoriasis patients. Journal of the European Academy of Dermatology and Venereology, 2012; 26 (7), pp. 833-837.

[24] Galassetti, P. Inflammation and oxidative stress in obesity, metabolic syndrome, and diabetes. Experimental Diabetes Research, 2012. Article ID 943706, 2 pages, doi: $10.1155 / 2012 / 943706$.

[25] Benedetti, S., Tagliamonte, M. C., Catalani, S., Primiterra, M., Canestrari, F., De Stefani, S., Palini, S. and Bulletti, C. Differences in blood and semen oxidative status in fertile and infertile men, and their relationship with sperm quality. Reproductive biomedicine online, 2012; 25 (3), pp. 300-306.

[26] Shamsi, M. B., Venkatesh, S., Kumar, R., Gupta, N. P., Malhotra, N., Singh, N., et al.,. Antioxidant levels in blood and seminal plasma and their impact on sperm parameters in infertile men. Indian journal of biochemistry \& biophysics, 2010; 47 (1), p. 38.

[27] Kayar, A., Dokuzeylul, B., Kandemir, F. M., Kirbas, A., Bayrakal, A. and Or, M. E. Total oxidant and antioxidant capacities, nitric oxide and malondialdehyde levels in cats seropositive for the feline coronavirus. Veterinarni Medicina, $2015 ; 60$ (5), pp. 274-281.

[28] Kose, O., Canakci, V., Canakci, C. F., Yildirim, A., Kermen, E., Arabaci, T. and Gungor, A. The effect of obesity on total antioxidant/oxidant status and oxidative stress index in patients with chronic periodontitis. Oxidants and Antioxidants in Medical Science, 2014; 3 (2), pp. 153-159. 